\title{
Computational Studies of Aromatic and Photophysical Properties of Expanded Porphyrins
}

\section{Valiev, Rashid R.}

2018-05-24

Valiev , R R , Benkyi , I , Konyshev , Y , Fliegl , H \& Sundholm , D 2018 , ' Computational Studies of Aromatic and Photophysical Properties of Expanded Porphyrins ', Journal of Physical Chemistry A , vol. 122 , no. 20 , pp. 4756-4767 . https://doi.org/10.1021/acs.jpca.8b02311

http://hdl.handle.net/10138/309481

https://doi.org/10.1021/acs.jpca.8b02311

unspecified

acceptedVersion

Downloaded from Helda, University of Helsinki institutional repository.

This is an electronic reprint of the original article.

This reprint may differ from the original in pagination and typographic detail.

Please cite the original version. 


\section{Computational studies of aromatic and}

\section{photophysical properties of expanded porphyrins}

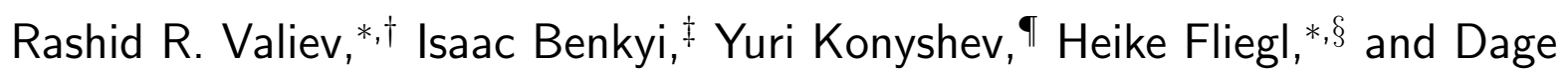
Sundholm*,\|

$\dagger$ University of Helsinki, Department of Chemistry, P.O. Box 55 (A.I. Virtanens plats 1), FIN-00014 University of Helsinki, Finland

and Tomsk Polytechnic University, 43a, Lenin Avenue, Building 2, 634050 Tomsk, Russian Federation

$\ddagger$ University of Helsinki, Department of Chemistry, P.O. Box 55 (A.I. Virtanens plats 1), FIN-00014 University of Helsinki, Finland.

\Tomsk State University, Lenina Avenue 36; Tomsk, Russian Federation

$\S$ Hylleraas Centre for Quantum Molecular Sciences, Department of Chemistry, University of Oslo, P.O.Box 1033 Blindern, 0315 Oslo, Norway

|| University of Helsinki, Department of Chemistry, P.O. Box 55 (A.I. Virtanens plats 1), FIN-00014 University of Helsinki, Finland

and Centre for Advanced Study at the Norwegian Academy of Science and Letters, Drammensveien 78, N-0271 Oslo, Norway.

E-mail: valievrashid@gmail.com; Heike.Fliegl@kjemi.uio.no; Dage.Sundholm@helsinki.fi 


\begin{abstract}
Magnetically induced current densities and ring-current pathways have been calculated at density functional theory (DFT) and second-order Møller-Plesset perturbation theory (MP2) levels of theory for a set of expanded porphyrins consisting of five or six pyrrolic rings. The studied molecules are sapphyrin, cyclo[6]pyrrole, rubyrin, orangarin, rosarin, and amethyrin. Different functionals have been employed to assess the functional dependence of the ring-current strength susceptibility. Vertical singlet and triplet excitation energies have been calculated at the second-order approximate coupled cluster (CC2), expanded multi-configurational quasi-degenerate perturbation theory (XMC-DPT2), and time-dependent density functional theory levels. The lowest electronic transition of the antiaromatic molecules were found to be pure magnetic transitions providing an explanation for the large paratropic contribution to the total current density. Rate constants for different nonradiative deactivation channels of the lowest excited states have been calculated yielding lifetimes and quantum yields of the lowest excited singlet and triplet states. The calculations show that the spin-orbit interaction between the lowest singlet $\left(S_{0}\right)$ and triplet $\left(T_{1}\right)$ states of the antiaromatic molecules is strong, whereas for the aromatic molecule the spin-orbit coupling vanishes. The experimentally detected fluorescence from $S_{2}$ to $S_{0}$ of amethyrin has been explained. The study shows that there are correlations between the aromatic character and optical properties of the investigated expanded porphyrins.
\end{abstract}

\title{
1 Introduction
}

Porphyrinoids are porphyrin derivatives with one or several of its four pyrrolic rings modified by inserting heteroatoms or by adding substituents. ${ }^{1}$ Their aromatic character depends on the number and properties of the heteroatoms and what kind of substituents that are conjugated with the main macrocycle. ${ }^{2-7}$ Many antiaromatic porphyrinoids are stable with interesting properties for technological devices. ${ }^{2,8,9}$ The term antiaromatic was used for the first time by Breslow in 1965. ${ }^{10,11}$ Some of the antiaromatic porphyrinoids have been found 
to possess high conductivity making them useful as building blocks in conductive materials. ${ }^{12,13}$ The aromatic character of porphyrinoids has therefore been investigated computationally in a number of works. ${ }^{14-28}$ Computational and experimental studies have revealed novel properties of antiaromatic porphyrinoids such as that closed-shell strongly antiaromatic porphyrinoids can be paramagnetic. ${ }^{29,30}$ Thus, one can expect that the magnetic properties of such molecules change when being exposed to a strong magnetic field. ${ }^{31,32}$ In a recent study, we showed that the paramagnetic properties of the antiaromatic porphyrinoids originate from a low-lying electronic dipole-forbidden singlet state with a strong magnetic-allowed transition. $^{33}$

The low-lying electronic state with electronic dipole-forbidden transitions may also be the underlying reason for the unusual photophysical properties of strongly antiaromatic expanded porphyrins, ${ }^{34,35}$ whose lowest electronic singlet state is reached by a magnetic-dipole allowed electronic transition. ${ }^{34,35}$ Experimental studies have shown that the antiaromatic expanded porphyrins have a very weak fluorescence or even lacking it. ${ }^{34,36} \mathrm{Kim}$ and coworkers measured the total lifetimes of low-lying singlet and triplet states of three aromatic and three antiaromatic expanded porphyrins. ${ }^{34,35}$ The study showed that the first triplet electronic state of the antiaromatic molecules decays rapidly to the ground state with the triplet-state lifetime in the nanosecond regime. The lifetime of the lowest singlet state of the antiaromatic porphyrinoids is up to 30 times shorter than for the aromatic ones, ${ }^{34}$ probably because of the optically dark state in the near infrared region (NIR) that participates in the relaxation dynamics process of the excited states. ${ }^{34}$

Recently, we investigated photophysical properties of classic porphyrins such as free-base porphyrin, tetraphenylporphyrins and tetrabenzoporphyrins using quantum chemical calculations. ${ }^{37}$ Rate constants for intramolecular nonradiative transitions such as internal conversion (IC) and intersystem crossing (ISC) were calculated for transitions between low-lying singlet and triplet states. The same computational approach has also been successfully employed in calculations of rate constants for nonradiative electronic transitions in [8]circulene 
derivatives. ${ }^{38-40}$

The aim of this work is to calculate rate constants for radiative and nonradiative electronic transitions between low-lying singlet and triplet states of expanded porphyrinoids. Calculations of magnetically induced current densities and current pathways provide additional information about relations between photophysical properties and the aromatic character of the studied molecules.

\section{Computational methods}

\subsection{Molecular structures}

The initial molecular structures were taken from Ref. 35 and were reoptimized with Turbomole version 7.2 at the density functional theory (DFT) level using the B3LYP functional ${ }^{41,42}$ and the Karlsruhe triple- $\zeta$ quality basis sets (def2-TZVP). ${ }^{43-46}$ The optimized structures of the expanded porphyrinoids are shown in 1. The Cartesian coordinates of the optimized molecular structures are given as Supporting Information. The notation for the irreducible representations of the symmetric molecules is used for labeling the excited states. The molecular structure of molecule III was also optimized using the M06-2X functional, because recent studies suggested that functionals with significant Hartree-Fock exchange are needed when studying expanded porphyrinoids. ${ }^{47-49}$ However, the optimized structures using the B3LYP and M06-2X functionals are almost identical with an RMS deviation of $0.35 \AA$ for the C-C bond distances. The Cartsian coordinates of the two structures are given as Supporting Information.

Sapphyrin (I) consists of five pyrrolic rings, which are connected via four methine bridges and one direct carbon-carbon link. The molecular structure of sapphyrin with three inner hydrogens belongs to the $\mathrm{C}_{2 v}$ point group. Cyclo[6]pyrrole (II) consists of six directly linked pyrrolic rings. It has four inner hydrogens and belongs to the $\mathrm{D}_{2 h}$ point group. Rubyrin (III) consists of six pyrrolic rings, which are connected via four methine bridges and two direct 


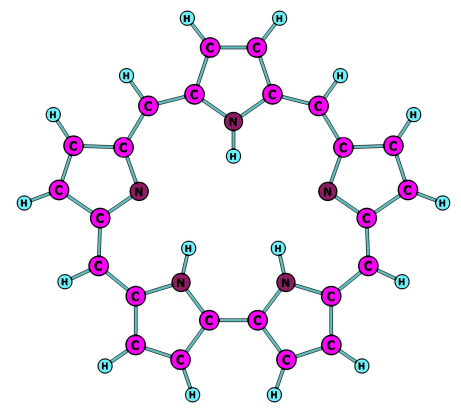

(I)

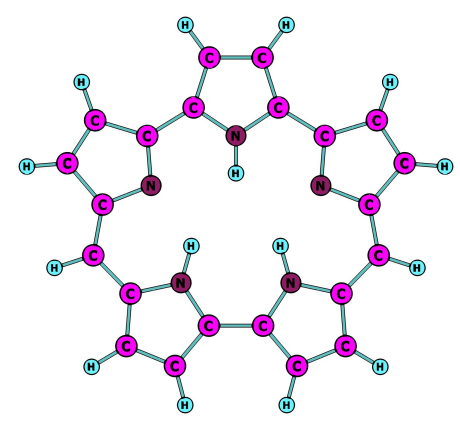

(IV)

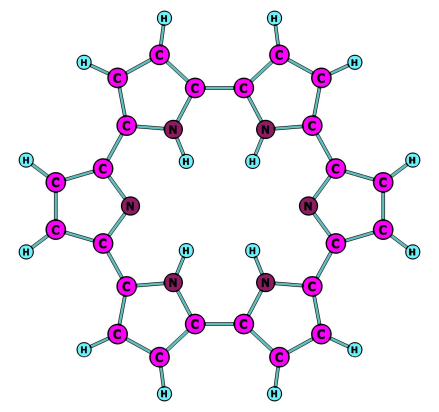

(II)

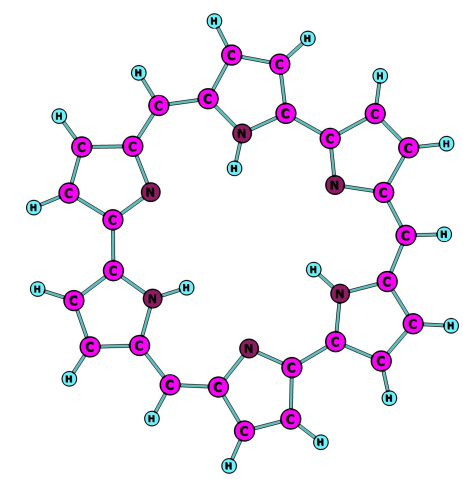

( V)

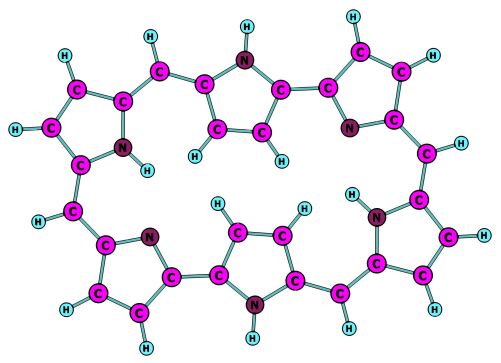

(III)

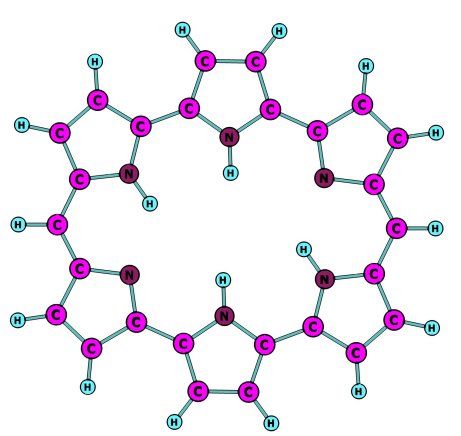

$(\mathrm{VI})$

Figure 1: The molecular structure of the studied expanded porphyrins. Carbon atoms are shown in pink, nitrogens are brown and hydrogens are light blue. 
carbon-carbon links. Two of the directly linked pyrrolic rings are inverted. The molecular structure of rubyrin with four hydrogens connected to the six pyrrolic nitrogen belongs to the $\mathrm{C}_{2 h}$ point group. Orangarin (IV) consists of five pyrrolic rings, which are connected via two methine bridges and three direct links. Orangarin with three inner hydrogens belongs to the $\mathrm{C}_{2 v}$ point group. Rosarin $(\mathrm{V})$ consists of six pyrrolic rings, which are connected via three alternating methine bridges and carbon-carbon links. Rosarin has three inner hydrogens and belongs to the $\mathrm{C}_{3 h}$ point group. Amethyrin (VI) consists also of six pyrrolic rings, which are connected via two methine bridges and four direct links. Amethyrin has four inner hydrogens and belongs to the $\mathrm{C}_{2 h}$ point group.

\subsection{Methods for calculating excitation energies}

The excited states of the expanded porphyrinoids were studied at ab initio levels of theory. Excitation energies were also calculated at the time-dependent density functional theory (TDDFT) level, even though previous calculations showed that TDDFT yields overestimated excitation energies for the lowest singlet states of the antiaromatic porphyrinoids. ${ }^{34,35}$

We have calculated the lowest singlet and triplet excitation energies at the expanded multi-configurational quasi-degenerate perturbation theory (XMC-QDPT2) level using the def2-TZVP basis sets. ${ }^{50}$ The complete active space self-consistent field (CASSCF) calculations consider 8 electrons in 8 molecular orbitals (MO) for molecules I, II, IV and VI, whereas for molecules III and VI the CASSCF space consisted of 10 electrons in 9 orbitals and 12 electrons in 11 orbitals, respectively. We performed state-average CASSCF (SA-CASSCF) calculations over the 9 lowest electronic states.

In the XMC-QDPT2 calculations, the effective Hamiltonian included 30 states. The oscillator strengths were also calculated at the XMC-QDPT2 level. At the SA-CASSCF

level, the considered states are orthogonal with a common set of active molecular orbitals (MO) leading to a straight forward analysis and comparison of the electronic properties of the studied electronic states. Calculations at the XMC-QDPT2 ${ }^{50}$ level of theory are 
more efficient as compared to the original CASPT2 ${ }^{51}$ and related NEVPT2 $2^{52}$ level of theory calculations. An important advantage of XMC-QDPT2 calculations is that they do not suffer from intruder-state problems. ${ }^{50}$ Excitation-energy calculations at the XMC-QDPT2 were carried out using the Firefly software. ${ }^{53}$

Vertical excitation energies have also been examined at the second-order approximate coupled cluster level $(\mathrm{CC} 2)^{54}$ using the resolution-of-the-identity approximation ${ }^{55-57}$ as im-

plemented in Turbomole ${ }^{46,58}$ version 7.2. We employed the reduced-virtual-space (RVS) approximation, where orbital energies above $70 \mathrm{eV}$ are neglected in the correlation calculation of the excitation energy. ${ }^{59}$ The triplet excitation energies of rosarin $(\mathrm{V})$ were calculated at the configuration interaction singles level with a perturbative treatment of the double excitations (CIS(D)), ${ }^{60}$ because the $\mathrm{CC} 2$ calculations suffered from technical difficulties. The def2-TZVP basis sets were employed in the calculations. ${ }^{44}$

The singlet excitation energies were also calculated at the TDDFT level using the B3LYP functional and the def2-TZVP basis sets as implemented in Turbomole 7.2. ${ }^{41,42,44,61,62}$

\subsection{Methods for calculating spin-orbit coupling interactions}

The matrix elements of the spin-orbit coupling interaction between the singlet and the triplet states were calculated using perturbation theory. The SA-CASSCF wavefunctions and the XMC-QDPT2 excitation energies of the electronic states were used as zero-order wavefunctions and excitation energies, respectively. The Breit-Pauli one-electron operator was used as perturbation operator. The calculations of the spin-orbit couplings were performed using GAMESS-US software version $2016 .{ }^{63}$

\subsection{Methods for calculating rate constants}

The ground-state equilibrium molecular structures were used in the calculations of the photophysical properties, since the Stokes shift is very small for the considered molecules. ${ }^{34,35}$ Stokes shifts are generally very small for classical porphyrins. ${ }^{37}$ 
The radiative rate constants from the $i$-th excited singlet state $\left(S_{i}\right)$ to the electronic ground state $\left(S_{0}\right)$ were obtained by applying the Strickler-Berg equation: ${ }^{64}$

$$
k_{r}=0.7 \cdot E^{2} \cdot f
$$

where $E$ is the transition energy between $S_{0}$ and $S_{i}, f$ is the corresponding oscillator strength. The Strickler-Berg equation can be used for estimating the fluorescence lifetime when the Stokes shift is small indicating that the difference between the molecular structures of the electronic ground and excited states is small, ${ }^{64}$ which is generally the case for porphyrins.

The internal conversion rates $\left(k_{I C}\right)$ between excited spin-singlet states $\left(S_{i} \rightarrow S_{i-k}\right.$, with $k=0,1, . . i-1)$ were calculated using the following expression: ${ }^{65,66}$

$$
k_{I C}=N_{X-H} \cdot \frac{\left|\left\langle\psi\left(S_{i}\right)|\Omega| \psi\left(S_{i-k}\right)\right\rangle\right|^{2}}{E^{2}} \cdot 10^{20-\frac{E}{5}}
$$

where $E$ the energy difference (in $\left.\mathrm{cm}^{-1}\right)$ between the $S_{i}$ and $S_{i-k}(k=1, . . i-1)$ states, $\mathrm{N}_{X-H}$ is the number of all $\mathrm{X}-\mathrm{H}$ bonds $(\mathrm{X}=\mathrm{C}$ or $\mathrm{N}),\left\langle\psi\left(S_{i}\right)|\Omega| \psi\left(S_{i-k}\right)\right\rangle\left(\mathrm{in}^{-1}\right)$ are the nonadiabatic coupling matrix elements (NACME). The expression for the dependence between the rate constant $\left(k_{I C}\right)$ and energy difference $E$ between two states with the same spin was derived by assuming that the energy difference $E\left(S_{i}\right)$ and $E\left(S_{i-k}\right)$ transfers into the vibrations of $\mathrm{X}-\mathrm{H}$ bonds due to their high frequency and large anharmonicity. ${ }^{65}$ Eq. (2) has an expected accuracy of about $6 \% .{ }^{65}$ The NACMEs were calculated using the incomplete neglect of differential overlap (INDO) method as described in Refs. 65 and 66.

The rate constants for the intersystem crossing between $S_{i}$ and the $j$-th triplet state $\left(T_{j}\right)$ were estimated using the following expression: ${ }^{66}$

$$
k_{S_{i} T_{j}}=10^{10} \cdot\left|\left\langle\psi\left(S_{i}\right)\left|H_{S O}\right| \psi\left(T_{j}\right)\right\rangle\right|^{2} \cdot \rho
$$

where $\left\langle\psi\left(S_{i}\right)\left|H_{S O}\right| \psi\left(T_{j}\right)\right\rangle$ are matrix elements of the spin-orbit coupling interaction operator in $\mathrm{cm}^{-1}$. For macrocyclic organic molecules like porphyrins and circulenes, the main 
contribution to the Franck-Condon factor originates from vibrational states with energies of about $1400 \mathrm{~cm}^{-1}$, which corresponds to a Huang-Rhys factor of $y \approx 0.3{ }^{38,66,67}$ The density of the final states $(\rho)$ is determined at zero temperature by using Franck-Condon factors that were estimated from an average vibrational mode whose energy is $1400 \mathrm{~cm}^{-1}$. The obtained semi-empirical expression for the density of states is

$$
\rho=F_{0 n}=\frac{y^{n} \exp (-y)}{n !}
$$

where, $n \approx \frac{E\left(S_{i}\right)-E\left(T_{j}\right)}{1400}$ and $y=0.3$ is the Huang-Rhys factor for the $1400 \mathrm{~cm}^{-1}$ mode. ${ }^{66}$

The total lifetime $\left(\tau\left(S_{i}\right)\right)$ of the $S_{i}$ state and its fluorescence quantum yield $\left(\phi_{f l}\left(S_{i}\right)\right)$ were obtained as

$$
\begin{gathered}
\tau\left(S_{i}\right)=\frac{1}{\sum k_{r}+\sum k_{I C}+\sum k_{I S C}} \\
\phi_{f l}\left(S_{i}\right)=\frac{k_{r\left(S_{i}\right)}}{\sum k_{r}+\sum k_{I C}+\sum k_{I S C}}
\end{gathered}
$$

Here, $\sum k_{I C}$ denotes the sum of the internal conversion rate constants from $S_{i}$ into all singlet states with lower energy. $\sum k_{I S C}$ denotes the sum of intersystem crossing rate constants from $S_{i}$ to all triplet states that are energetically below $S_{i} \cdot k_{r\left(S_{i}\right)}$ is the radiative rate constant from $S_{i} \rightarrow S_{0}$. $\sum k_{r}$ denotes the sum of all radiative rate constants from $S_{i}$ to all singlet states that are energetically below $S_{i}$.

The phosphorescence rate constant $\left(k_{\text {phos }}\right)$ and the internal conversion rate constant $\left(k_{I C}\left(\widetilde{T}_{1} \rightarrow S_{0}\right)\right)$ from the spin-mixed first triplet state $\left(\widetilde{T}_{1}\right)$ to the singlet ground state $\left(S_{0}\right)$ were calculated using Eq. (7) and Eq. (8). ${ }^{68}$

$$
k_{\text {phos }}=\sum_{p} k_{r}\left(\left(S_{p} \rightarrow S_{0}\right)\right)\left|a_{p}\right|^{2}\left(\frac{E\left(T_{1}\right)}{E\left(S_{p}\right)}\right)^{3}+\sum_{m}\left|b_{m}\right|^{2} \cdot k_{r}^{m}\left(T_{m} \rightarrow T_{1}\right)\left(\frac{E\left(T_{1}\right)}{E\left(T_{1}\right)-E\left(T_{m}\right)}\right)^{3}
$$




$$
k_{I C}\left(\widetilde{T}_{1} \rightarrow S_{0}\right)=\sum_{p} k_{I C}\left(S_{p} \rightarrow S_{0}\right)\left|a_{p}\right|^{2}\left(\frac{E\left(T_{1}\right)}{E\left(S_{p}\right)}\right)^{2} \cdot \exp \left(\frac{\left(E\left(S_{0}\right)-E\left(T_{1}\right)\right)}{5}\right)
$$

where $a_{p}=\frac{\left\langle\psi\left(T_{1}\right)\left|H_{\mathrm{SO}}\right| \psi\left(S_{p}\right)\right\rangle}{E\left(T_{1}\right)-E\left(S_{p}\right)}$ and $b_{m}=\frac{\left\langle\psi\left(S_{0}\right)\left|H_{\mathrm{SO}}\right| \psi\left(T_{m}\right)\right\rangle}{E\left(S_{0}\right)-E\left(T_{m}\right)} . \widetilde{T}_{1}$ is a spin-mixed triplet state with a portion of singlet $\left(\psi\left(S_{p}\right)\right)$ character due to the spin-orbital interaction. $k_{r}^{m}\left(T_{m} \rightarrow T_{1}\right)$ is the radiative rate constant for the transition from $T_{m}$ to $T_{1}$. The wavefuction of the spin-mixed state can be expressed as $\psi\left(\widetilde{T}_{1}\right)=\psi\left(T_{1}\right)+\sum a_{p} \psi\left(S_{p}\right)$. The wavefunction of spinmixed singlet ground state has also some triplet state character $\psi\left(\widetilde{S}_{0}\right)=\psi\left(S_{0}\right)+\sum b_{m} \psi\left(T_{m}\right)$ implying that IC processes between $\widetilde{T}_{1}$ and $\widetilde{S}_{0}$ can also occur.

The rate constants $k_{r}^{m}\left(T_{m} \rightarrow T_{1}\right)$ estimated using Eq. (1) are large implying that contributions from higher triplet states to the overall $k_{\text {phos }}$ rate constant can be omitted.

The lifetime of the lowest triplet state was obtained as

$$
\tau\left(T_{1}\right)=\frac{1}{k_{p h o s}+k_{i c}\left(\widetilde{T}_{1} \rightarrow S_{0}\right)+k_{T_{1} S_{0}}}
$$

\subsection{Methods for current density calculations}

The magnetically induced current densities were calculated at the DFT level using different functionals as well as at the second-order Møller-Plesset perturbation theory (MP2) level. Current densities were calculated using the GIMIC program, ${ }^{69-72}$ which is an independent program that uses basis-set information and density matrices in atomic orbital basis as input data. The first-order magnetically perturbed density matrices are obtained from nuclear magnetic shielding calculations. ${ }^{69,70}$ GIMIC calculations performed using def2-TZVP basis sets $^{44,73}$ yield accurate gauge-origin independent current densities, because gauge-including atomic orbitals (GIAO), a.k.a. London atomic orbitals (LAO) are employed. ${ }^{74,75}$ The nuclear magnetic resonance (NMR) shieldings were calculated at the DFT and MP2 levels using the MPSHIFT module of Turbomole. ${ }^{76,77}$ The B3LYP, ${ }^{41,42}$ BHLYP, ${ }^{78}$ PBE0, ${ }^{79}$ TPSS, ${ }^{80}$ TPSSh $^{81}$ 
and M06-2X ${ }^{82}$ functionals were employed in the magnetic-shielding and current-density calculations in order to assess which functional works well in calculations of magnetic properties of antiaromatic porphyrinoids. The magnetically induced current densities were also calculated at the MP2 level to check the reliability of the employed functionals, ${ }^{77,83}$ since previous current density calculations on strongly antiaromatic porphyrinoids showed that B3LYP calculations exaggerate the paratropic ring current strength around the macroring. ${ }^{29} \mathrm{~A}$ recent overview of current density studies on porphyrinoids can be found in Ref. 28 .

\section{Results and discussion}

\subsection{Current-density calculations}

The current-density calculations confirm the aromatic character of the expanded porphyrins that were deduced form experimental data. ${ }^{34}$ The signed modulus of the current-density are plotted in 2 and ring-current strengths calculated at different levels of theory are summarized in 1 . We recently showed that there is a linear correlation between the magnetic and energetic aromaticity criteria, ${ }^{84}$ implying that the ring-current criterion can be used for assessing the aromatic character. The ring-current strengths for the aromatic expanded porphyrins (molecules I, II and III) calculated at the B3LYP level are in the range of 24-30 nA/T, which is of the same size as the ones obtained for the classical porphyrins. ${ }^{19}$ Molecules IV, V and VI sustain strong paratropic ring currents implying that they are antiaromatic according to the ring-current criterion.

Ring-current strengths calculated at different levels of theory are compared in 1 . The calculations show that roughly the same ring current strengths are obtained for the aromatic molecules II and III at all employed levels of theory. The ring-current strength was also calculated for molecule III at the M06-2X level using the molecular structure optimized at the same level. The obtained ring-current strength differ by $1 \%$ from the one obtained with the M06-2X functional using the molecular structure optimized at the B3LYP level. 
For molecule I, ring-current strengths calculated using different DFT functionals agree well, whereas MP2 calculations yield a ring-current strength of $50 \mathrm{nA} / \mathrm{T}$, which is $20 \mathrm{nA} / \mathrm{T}$ larger than the one obtained at the other levels of theory. Judged from the multireference diagnostics, molecule I seems to be dominated by one reference determinant. The calculated excitation energies are as accurate as the ones obtained for molecules II and III. The reason for the large correlation contribution to the ring-current strength at the MP2 level of theory could not been elucidated, since current-density calculations at higher levels of theory are not feasible for molecules of this size.

For the antiaromatic molecules, calculations at the DFT levels overestimate the ringcurrent strength, when assuming that the MP2 values are the most accurate ones. Hybrid functionals seem to work better than pure DFT functionals suggesting that the incorrect shape of the long-ranged potential leads to a strong paratropic ring current. The electron mobility is reduced by introducing HF exchange in the functional. The best agreement with the MP2 values was obtained when using the BHLYP functional that has $50 \%$ HF exchange. The deviation from the MP2 values increases with decreasing amount of HF exchange in the functional. The M06-2X functional with 54\% HF exchange slightly underestimates the ringcurrent strengths of the antiaromatic porphyrinoids. The ring-current strengths calculated at the HF level are about a factor of two smaller than the ones obtained at the MP2 level.

The current strengths passing individual bond shown in 3 yield the pathways of the ring currents. The calculations show that the ring-current splits for all the studied molecules at the pyrrolic rings. Thus, the current pathways of the expanded porphyrins are very similar to those obtained for other porphyrinoids. ${ }^{28}$ There is no evidence for strong local currents in the pyrrolic rings suggesting that all $\pi$-electrons participate in the aromatic pathways as also obtained for other porphyrinoids. ${ }^{28}$

Calculations at the B3LYP level show that sapphyrin (I) sustains a net ring-current strength susceptibility of $30.1 \mathrm{nA} / \mathrm{T}$. The current strengths in 1 and 3 slightly differ, because different integration grids and planes have been used. The obtained current strengths at the 


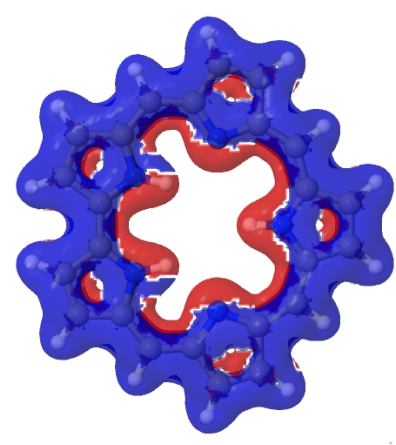

(I)

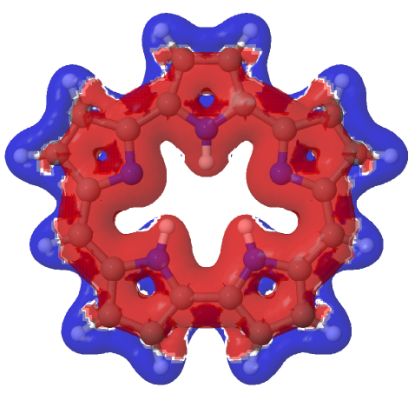

(IV)

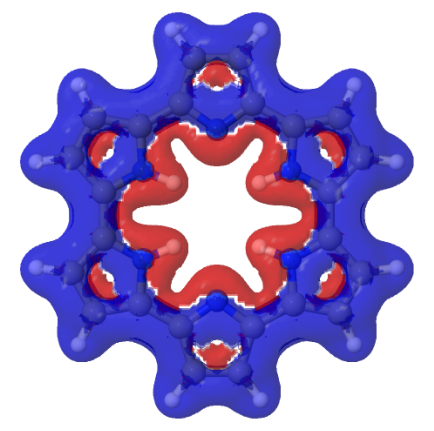

(II)

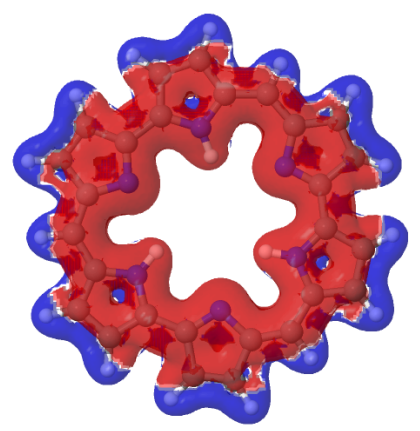

$(\mathrm{V})$

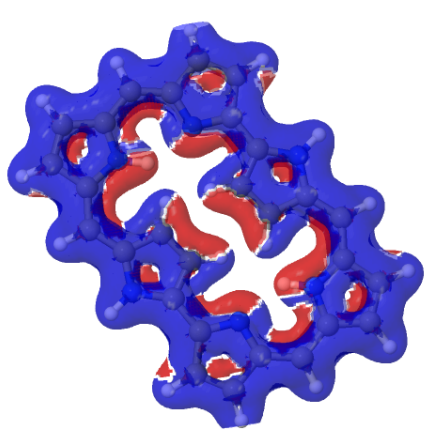

(III)

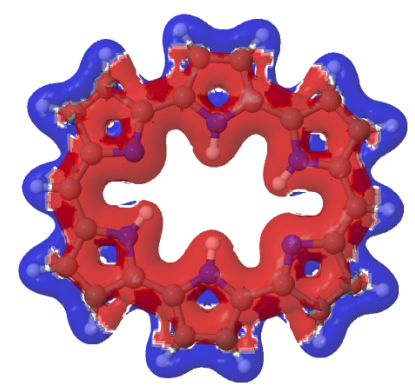

(VI)

Figure 2: The signed modulus of the calculated current densities for molecules I-VI. The modulus of the paratropic current density is shown in red and the diatropic contribution is blue.

Table 1: The net ring-current strength (in $\mathrm{nA} / \mathrm{T}$ ) around the porphyrin macroring of molecules I-VI calculated at the Hartree-Fock (HF), the second-order Møller-Plesset (MP2) and density functional theory (DFT) levels of theory.

\begin{tabular}{lrrrrrrrr}
\hline Molecule & B3LYP & BHLYP & PBE0 & TPSS & TPSSh & M06-2X & HF & MP2 \\
\hline I & 28.2 & 30.5 & 29.9 & 28.5 & 29.3 & 32.8 & 25.4 & 50.5 \\
\hline II & 23.5 & 26.1 & 24.1 & 22.2 & 23.0 & 27.6 & 29.2 & 23.5 \\
\hline III & 28.9 & 30.0 & 28.8 & 28.0 & 27.5 & 32.7 & 32.9 & 29.3 \\
\hline IV & -36.1 & -25.9 & -34.5 & -40.4 & -37.8 & -21.5 & -14.0 & -30.3 \\
\hline V & -39.7 & -26.5 & -37.6 & -43.8 & -42.3 & -23.5 & -12.8 & -25.8 \\
\hline VI & -30.9 & -21.0 & -29.3 & -36.5 & -33.5 & -19.0 & -9.5 & -22.8 \\
\hline
\end{tabular}




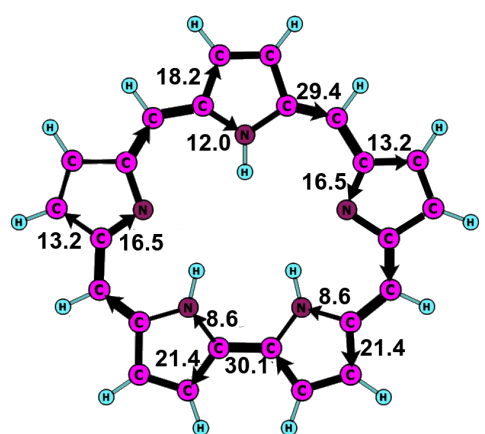

(I)

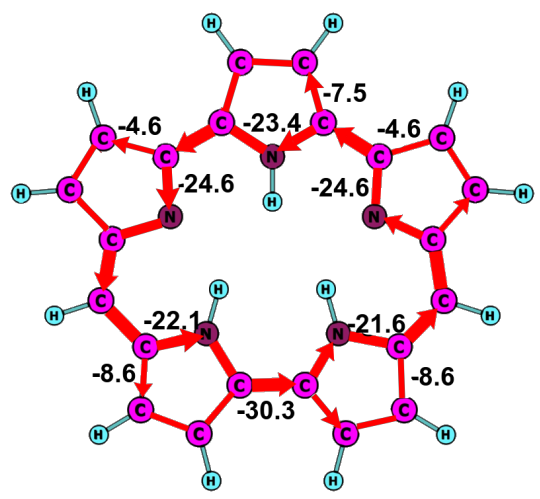

(IV)

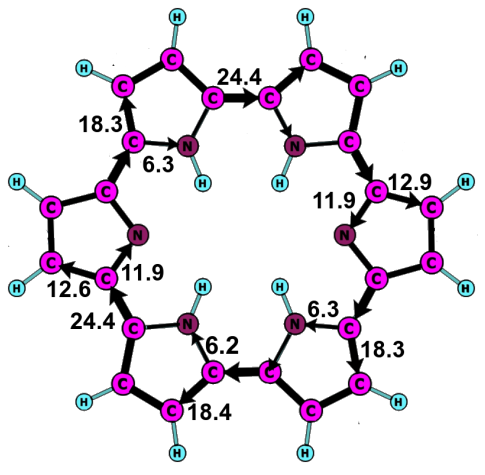

(II)

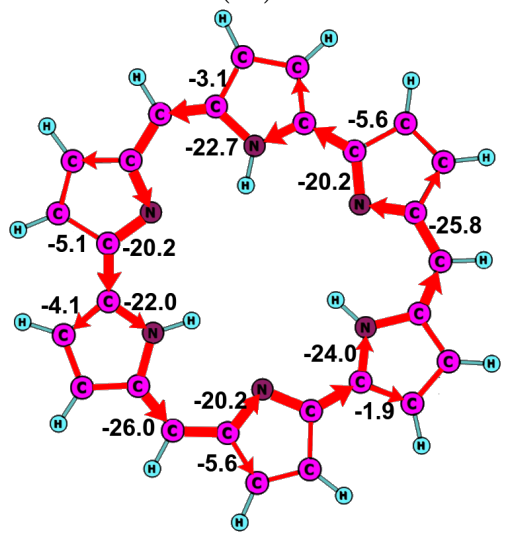

(V)

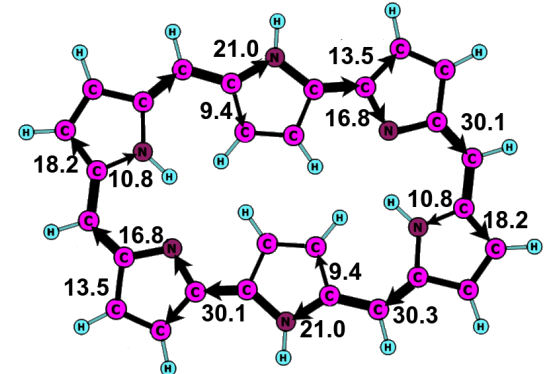

(III)

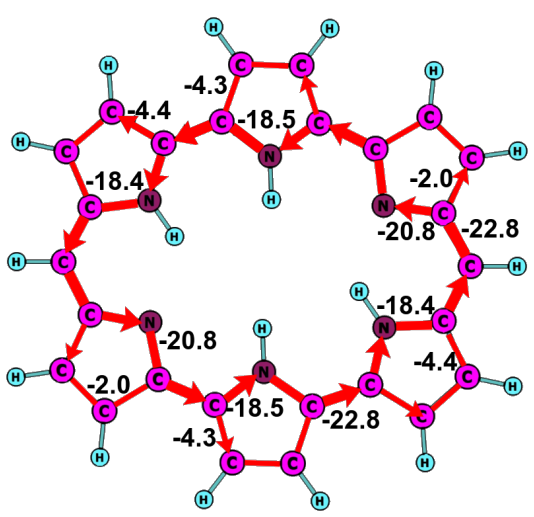

(VI)

Figure 3: The net ring-current strengths (in $\mathrm{nA} / \mathrm{T}$ ) passing selected bonds in the aromatic molecules I-III. The current densities of I-III were calculated at the B3LYP/def2-TZVP level. The current pathways of the aromatic molecules are indicated by black arrows. For the antiaromatic molecules (IV-VI), the ring-current strengths and pathways were calculated at the MP2/def2-TZVP level. The current pathways of the antiaromatic molecules (IV-VI) are indicated by red arrows. 
DFT levels almost the same indicating that molecule I is aromatic according to the ringcurrent criterion. The ring current divides into an outer and inner pathway at each pyrrolic ring as it in general does for porphyrinoids. ${ }^{28}$ For pyrrolic rings with an inner hydrogen, the main part of the ring current takes the outer route via the $\beta$ carbons. At the two neighbouring pyrrolic rings that are directly connected via a C-C bond, a current of 21.4 $\mathrm{nA} / \mathrm{T}$ flows via the $\beta$ carbons and $8.6 \mathrm{nA} / \mathrm{T}$ passes the $\mathrm{NH}$ moiety. The third pyrrolic ring with an inner hydrogen sustains current of $18.2 \mathrm{nA} / \mathrm{T}$ along the outer route and $12.0 \mathrm{nA} / \mathrm{T}$ passes via the NH moiety. For the two pyrrolic rings without an inner hydrogen, the current strength along the inner pathway of $16.5 \mathrm{nA} / \mathrm{T}$ is slightly stronger than the one of $13.5 \mathrm{nA} / \mathrm{T}$ passing the $\beta$ carbons.

Cyclo[6]pyrrole (II) is aromatic with a ring-current strength susceptibility of $24.4 \mathrm{nA} / \mathrm{T}$ at the B3LYP level. The pyrrolic rings with an inner hydrogen sustain a current flow of 18.4 $\mathrm{nA} / \mathrm{T}$ along the outer route and a current of $6.2 \mathrm{nA} / \mathrm{T}$ passes via the $\mathrm{NH}$ moiety. For the pyrrolic rings without an inner hydrogen, the ring current is almost equally divided between the two pathways.

Rubyrin (III) is aromatic sustaining a ring-current strength of $30.1 \mathrm{nA} / \mathrm{T}$ at the B3LYP level. For the pyrrolic rings without an inner hydrogen the ring current divides into two almost equally strong pathways. The current strength of the inner pathway is $16.8 \mathrm{nA} / \mathrm{T}$ and a current of $13.5 \mathrm{nA} / \mathrm{T}$ flows via the $\beta$ carbons. The current strength of the outer pathway of the rings with an inner hydrogen is $18.2 \mathrm{nA} / \mathrm{T}$ and the current strength for the inner one is $10.8 \mathrm{nA} / \mathrm{T}$. A strong current of $21.0 \mathrm{nA} / \mathrm{T}$ passes the $\mathrm{NH}$ moiety of the inverted pyrrolic rings, whereas $9.4 \mathrm{nA} / \mathrm{T}$ passes the $\beta$ carbons on the inside of the porphyrinoid ring. The ring-current strength of rubyrin is $10 \%$ stronger than for free-base porphyrin, whereas the porphyrinoids consisting of free-base porphyrin with one or two inverted pyrrolic rings sustain ring currents of only 13.0-16.4 nA/T. ${ }^{22}$

Organarin (IV) is antiaromatic sustaining a net current strength of $-36.1 \mathrm{nA} / \mathrm{T}$ at the B3LYP level. MP2 calculations yield a ring-current strength of $-30.3 \mathrm{nA} / \mathrm{T}$. Since B3LYP 
might overestimate the ring-current strengths of the antiaromatic porphyrinoids, the MP2 values are used in the analysis of the current pathways. The current strengths of the inner pathways are much stronger than the outer ones. Paratropic currents of $-21.6 \mathrm{nA} / \mathrm{T}$ to -24.6 take the inner route at the pyrrolic rings, whereas the current strengths via the $\beta$ carbons are in the range of $-4.6 \mathrm{nA} / \mathrm{T}$ to $-8.6 \mathrm{nA} / \mathrm{T}$. Thus, the ring current splits into two pathways at all pyrrolic rings. The current strength of the inner pathway is somewhat weaker when the nitrogen has an inner hydrogen.

Rosarin (V) is antiaromatic sustaining a current strength of $-26.0 \mathrm{nA} / \mathrm{T}$ at the MP2 level. The ring current flows mainly along the inner pathway at the pyrrolic rings. The current strengths via the $\beta$ carbons are in the range of $-1.9 \mathrm{nA} / \mathrm{T}$ to $-5.6 \mathrm{nA} / \mathrm{T}$. The ring current splits at the pyrrolic rings and no local ring currents in the pyrrolic rings are observed.

Amethyrin (VI) is antiaromatic sustaining a current strength of $-22.8 \mathrm{nA} / \mathrm{T}$ at the MP2 level. The paratropic current flow along inner pathway dominates. The current strengths via the $\beta$ carbons are in the range of $-2.0 \mathrm{nA} / \mathrm{T}$ to $-4.4 \mathrm{nA} / \mathrm{T}$. The current flow is slightly stronger along the inner route for pyrrolic rings without an inner hydrogen.

\subsection{Electronic excitation energies}

Calculated and experimental values for the lowest singlet excitation energies and the oscillator strengths of the studied molecules are compared in 2. The first singlet excitation energies calculated at the XMC-QDPT2 are about $0.2 \mathrm{eV}$ smaller than the experimental values, whereas CC2 and B3LYP excitation energies are 0.2-0.3 eV larger than the experimental ones for the aromatic porphyrinoids. Comparison of calculated excitation energies shows that the lowest CC2 and B3LYP excitation energies of the aromatic molecules are 0.4-0.5 eV larger than those obtained at the XMC-QDPT2 level of theory. Experimental

values for the $S_{2}$ state have not been reported. The CC2 excitation energies are 0.2-0.5 eV larger than the XMC-QDPT2 ones, whereas the B3LYP and XMC-QDPT2 excitation energies agree better with deviations of $0.0-0.3 \mathrm{eV}$. 
Table 2: Vertical excitation energies (in eV) of the lowest singlet states calculated at the XMC-QDPT2 and CC2 levels of theory are compared with experimental data. Excitation energies calculated at the TDDFT level using the B3LYP functional are also reported. The oscillator strengths are given in parenthesis. The molecular point groups and the irreducible representation of the excited states are also given. Experimental data are taken from Refs. 34 and 35.

\begin{tabular}{|c|c|c|c|c|c|c|}
\hline Level/Molecule & $\mathrm{I}\left(\mathrm{C}_{2 v}\right)$ & II $\left(\mathrm{D}_{2 h}\right)$ & III $\left(\mathrm{C}_{2 h}\right)$ & IV $\left(\mathrm{C}_{2 v}\right)$ & $\mathrm{V}\left(\mathrm{C}_{3 h}\right)$ & $\mathrm{VI}\left(\mathrm{C}_{2 h}\right)$ \\
\hline XMC-QDPT2 & $1.54(0.0002)$ & $1.34(0.50)$ & $1.14(0.04)$ & $1.19(0.00)$ & $1.24(0.00)$ & $1.25(0.00)$ \\
\hline $\mathrm{CC} 2$ & $1.98(0.002)$ & $1.88(0.33)$ & $1.53(0.06)$ & $1.23(0.01)$ & $1.35(0.00)$ & $1.32(0.00)$ \\
\hline B3LYP & $1.97(0.002)$ & $1.84(0.28)$ & $1.49(0.04)$ & $0.96(0.00)$ & $0.90(0.00)$ & $1.00(0.00)$ \\
\hline$S_{1}$ Exp. & $1.74 \quad \mathrm{~A}_{1}$ & $1.56 \quad \mathrm{~B}_{2 u}$ & $1.34 \quad \mathrm{~B}_{u}$ & $-\quad B_{1}$ & $-\quad \mathrm{A}^{\prime}$ & $\mathrm{A}_{g}$ \\
\hline XMC-QDPT2 & $1.91(0.04)$ & $1.56(0.85)$ & $1.69(0.03)$ & $2.10(0.03)$ & $2.05(0.00)$ & $2.12(0.80)$ \\
\hline $\mathrm{CC} 2$ & $2.27(0.05)$ & $2.05(0.52)$ & $1.90(0.03)$ & $2.87(0.45)$ & $3.01(0.04)$ & $2.76(0.65)$ \\
\hline B3LYP & $2.05(0.02)$ & $1.87(0.40)$ & $1.69(0.02)$ & $2.46(0.12)$ & $2.39(0.00)$ & $2.27(0.10)$ \\
\hline$S_{2}$ Exp. & $-\quad \mathrm{B}_{1}$ & $-\quad \mathrm{B}_{3 u}$ & $-\quad \mathrm{B}_{u}$ & $2.25 \quad \mathrm{~A}_{1}$ & $2.24 \quad \mathrm{E}^{\prime}$ & $2.08 \mathrm{~B}_{u}$ \\
\hline
\end{tabular}

For the antiaromatic molecules, the CC2, B3LYP and XMC-QDPT2 excitation energies of the $S_{1}$ state agree well suggesting that the $S_{1}$ state can be described by single electron replacements from the ground state configuration. The CC2 excitation energies are 0.04$0.11 \mathrm{eV}$ larger than the XMC-QDPT2 values, whereas B3LYP slightly underestimate the excitation energies with deviations of $0.25-0.35 \mathrm{eV}$ with respect to XMC-QDPT2 energies. The CC2 excitation energies of the $S_{2}$ state are $0.6-1.0 \mathrm{eV}$ larger than the ones calculated at the XMC-QDPT2 level. The large difference between the values calculated at the two levels of theory and the deviation of $0.62-0.75 \mathrm{eV}$ indicate that the $S_{2}$ states have significant multiconfiguration character. The $S_{2}$ energies calculated at the B3LYP level agree better with experimental data having excitation energies that are 0.14-0.20 eV larger than the experimental values. Calculations at the XMC-QDPT2 level yield $S_{2}$ excitation energies that deviate by $0.04-0.19 \mathrm{eV}$ from the experimental ones.

The lowest triplet excitation energies calculated at the XMC-QDPT2 and CC2 levels are compared in 3 . For rosarin $(\mathrm{V})$ with $\mathrm{C}_{3 h}$ symmetry, we were not able to converge the $\mathrm{CC} 2$ equations for the $T_{2}$ to $T_{4}$ states. The CIS(D) excitation energies of rosarin are therefore reported in 3. The calculations yielded on the average $0.4 \mathrm{eV}$ lower triplet excitation energies at the XMC-QDPT2 level than obtained in the $\mathrm{CC} 2$ calculations. The three lowest CIS(D) 
excitation energies of rosarin agree well with the XMC-QDPT2 values, whereas the fourth CIS(D) excitation energy is $0.85 \mathrm{eV}$ larger than obtained at the XMC-QDPT2 level.

The $S_{0} \rightarrow S_{1}$ electronic transition of orangarin (IV) has the largest coefficient for the $\operatorname{HOMO}\left(7 \mathrm{a}_{2}\right)-\mathrm{LUMO}\left(9 \mathrm{~b}_{2}\right)$ transition. HOMO and LUMO have a nodal symmetry corresponding the angular quantum number $\lambda=5$. According to the molecular orbital rule, transitions with $\Delta \lambda=0$ lead to the paratropic global current,${ }^{14}$ which is also obtained for orangarin (IV) in this work. The magnetic contribution to the transition probability dominates as for other antiaromatic porphyrinoids, ${ }^{33}$ since according to the selection rules for transition matrix elements over the angular momentum operator, the lowest singlet transition of the antiaromatic molecules (IV - VI) is a magnetic dipole transition. Thus, the antiaromatic character of molecules IV-VI is strongly influenced by the magnetically allowed $S_{0} \rightarrow S_{1}$ transition. Porphyrinoids with a strong magnetic dipole allowed $S_{0} \rightarrow S_{1}$ transition can be expected to be antiaromatic, because the lowest excitation will give rise to a large paratropic ring-current contribution as recently shown for antiaromatic carbaporphyrins and isophlorins. ${ }^{33}$ The reason for the fluorescent $S_{2} \rightarrow S_{0}$ state of the antiaromatic amethyrin (VI) is discussed below.

\subsection{Spin-orbit coupling}

The spin-orbit coupling interaction between the lowest triplet $T_{1}$ and ground state $S_{0}$ must vanish for molecules II and III due to the selection rules of the angular momentum for $D_{2 h}$ and $C_{2 h}$ symmetries. ${ }^{85}$ However, for molecules I and IV-VI, the selection rules for the transition matrix elements over the angular momentum operator allow $T_{1} \rightarrow S_{0}$ transition due to spin-orbit coupling, which might be the reason for the fast decay of the $T_{1}$ state for molecules IV-VI. The calculated matrix elements of the spin-orbit coupling operator in 4 confirm that the antiaromatic molecules have large spin-orbit coupling between the $T_{1}$ and $S_{0}$ states, whereas the matrix element vanishes for the aromatic ones.

The large non-zero $\left\langle\psi\left(S_{0}\right)\left|H_{S O}\right| \psi\left(T_{1}\right)\right\rangle$ matrix element for molecules IV, V and VI sug- 
Table 3: Excitation energies (in eV) of the lowest triplet states calculated at the XMC-QDPT2 and CC2 level of theory. For molecule V, the excitation energies of the triplet states were calculated at the CIS(D) level. The molecular point groups and the irreducible representation of the excited states are also given.

\begin{tabular}{llllllll}
\hline \hline Molecule & Level & $T_{1}$ & $T_{2}$ & $T_{3}$ & $T_{4}$ \\
\hline $\mathrm{I}\left(\mathrm{C}_{2 v}\right)$ & XMC-QDPT2 & $\mathrm{B}_{1}: 1.09$ & $\mathrm{~A}_{1}: 1.56$ & $\mathrm{~A}_{1}: 1.78$ & $\mathrm{~B}_{2}: 1.84$ \\
& CC2 & $\mathrm{B}_{1}: 1.63$ & $\mathrm{~A}_{1}: 1.96$ & $\mathrm{~A}_{1}: 2.13$ & $\mathrm{~B}_{1}: 2.23$ \\
$\mathrm{II}\left(\mathrm{D}_{2 h}\right)$ & XMC-QDPT2 & $\mathrm{B}_{1 u}: 0.58$ & $\mathrm{~B}_{2 u}: 0.94$ & $\mathrm{~B}_{3 g}: 1.82$ & $\mathrm{~A}_{g}: 2.10$ \\
& CC2 & $\mathrm{B}_{3 u}: 1.03$ & $\mathrm{~B}_{2 u}: 1.36$ & $\mathrm{~B}_{1} \mathrm{~g}: 2.62$ & $\mathrm{~B}_{2 u}: 2.79$ \\
$\mathrm{III}\left(\mathrm{C}_{2 h}\right)$ & XMC-QDPT2 & $\mathrm{B}_{u}: 0.84$ & $\mathrm{~B}_{u}: 1.06$ & $\mathrm{~B}_{u}: 1.24$ & $\mathrm{~B}_{u}: 1.80$ \\
& CC2 & $\mathrm{B}_{u}: 1.24$ & $\mathrm{~B}_{u}: 1.56$ & $\mathrm{~B}_{u}: 1.62$ & $\mathrm{~B}_{u}: 2.17$ \\
IV $\left(\mathrm{C}_{2 v}\right)$ & XMC-QDPT2 & $\mathrm{B}_{2}: 0.74$ & $\mathrm{~A}_{1}: 1.73$ & $\mathrm{~B}_{2}: 2.47$ & $\mathrm{~A}_{1}: 2.60$ \\
& CC2 & $\mathrm{B}_{1}: 1.02$ & $\mathrm{~A}_{1}: 2.02$ & $\mathrm{~B}_{1}: 2.53$ & $\mathrm{~A}_{1}: 2.91$ \\
$\mathrm{~V}\left(\mathrm{C}_{3 h}\right)$ & XMC-QDPT2 & $\mathrm{A}^{\prime}: 0.75$ & $\mathrm{E}^{\prime}: 1.74$ & $\mathrm{E}^{\prime}: 1.74$ & $\mathrm{~A}^{\prime}: 1.85$ \\
& CIS(D) & $\mathrm{A}^{\prime}: 0.86^{a}$ & $\mathrm{~A}^{\prime}: 1.61$ & $\mathrm{~A}^{\prime}: 1.61$ & $\mathrm{~A}^{\prime}: 2.70$ \\
$\mathrm{VI}\left(\mathrm{C}_{2 h}\right)$ & XMC-QDPT2 & $\mathrm{A}_{g}: 0.79$ & $\mathrm{~B}_{u}: 1.56$ & $\mathrm{~B}_{u}: 2.40$ & $\mathrm{~B}_{u}: 2.50$ \\
& CC2 & $\mathrm{A}_{g}: 1.12$ & $\mathrm{~B}_{u}: 1.90$ & $\mathrm{~B}_{u}: 2.50$ & $\mathrm{~A}_{g}: 3.05$ \\
\hline \hline
\end{tabular}

$a$ The CC2 excitation energy is $1.03 \mathrm{eV}$.

Table 4: The matrix elements of the spin-orbit coupling interaction (in $\mathrm{cm}^{-1}$ ) between the singlet and triplet states calculated at the multiconfiguration selfconsistent field (MCSCF) level using the XMC-QDPT2 excitation energies.

\begin{tabular}{lllllll}
\hline \hline Matrix element & I & II & III & IV & V & VI \\
\hline$\left\langle\psi\left(S_{0}\right)\left|H_{S O}\right| \psi\left(T_{1}\right)\right\rangle$ & 0.00 & 0.00 & 0.00 & 2.24 & 1.90 & 2.20 \\
$\left\langle\psi\left(S_{1}\right)\left|H_{S O}\right| \psi\left(T_{1}\right)\right\rangle$ & 3.00 & 2.95 & 2.91 & 0.00 & 1.18 & 0.03 \\
$\left\langle\psi\left(S_{1}\right)\left|H_{S O}\right| \psi\left(T_{2}\right)\right\rangle$ & 0.00 & 0.00 & 0.00 & 1.20 & 2.20 & 0.00 \\
$\left\langle\psi\left(S_{2}\right)\left|H_{S O}\right| \psi\left(T_{1}\right)\right\rangle$ & 0.00 & 0.00 & 0.00 & 2.40 & 0.00 & 0.00 \\
$\left\langle\psi\left(S_{2}\right)\left|H_{S O}\right| \psi\left(T_{2}\right)\right\rangle$ & 0.00 & 0.00 & 1.80 & 0.00 & 1.50 & 0.00 \\
$\left\langle\psi\left(S_{2}\right)\left|H_{S O}\right| \psi\left(T_{3}\right)\right\rangle$ & 0.00 & 0.00 & 0.00 & 0.00 & 1.60 & 0.00 \\
$\left\langle\psi\left(S_{2}\right)\left|H_{S O}\right| \psi\left(T_{4}\right)\right\rangle$ & 3.30 & 0.00 & 0.00 & 0.00 & 0.00 & 0.00 \\
\hline \hline
\end{tabular}


gests that intersystem crossing is fast, when also the energy difference between $T_{1}$ and $S_{0}$ states is small. The rate constant for phosphorescence $\left(k_{p h o s}\right)$ and the one for intersystem crossing $\left(k_{I C}\left(\widetilde{T}_{1} \rightarrow S_{0}\right)\right)$ cannot be expected to be small enough to compete with the singlettriplet rate constant $\left(k_{I S C}\right)$ for the decay process of the $T_{1}$ state. All rate constants involved in the excitation-energy deactivation process have to be calculated for obtaining a detailed understanding of the reasons for the fast triplet decay.

\subsection{Rate constants, lifetimes and quantum yields}

The calculated rate constants for the investigated deactivation channels of the $S_{1}, S_{2}$ and $T_{1}$ states of molecules I-VI are summarized in 4 . The rate constants were obtained by using the spin-orbit coupling matrix elements listed in 4, the oscillator strengths and excitation energies calculated at the XMC-QDPT2 level given in 2 and 3, and the nonadiabatic coupling matrix elements (NACME) calculated at the INDO level.

The rate of the IC process dominates the decay of the $S_{2}$ state for all molecules. The IC rate from $S_{1}$ to $S_{0}$ is also fast for all molecules. For molecule I, the triplet manifold is significantly occupied via the ISC process from $S_{2}$ to $T_{4}$ and from $S_{1}$ to $T_{1}$. The calculated rate constant for the ISC processes from the $S_{2}$ state of molecule I is only a factor of ten smaller than the IC rate costant of the $S_{2}$ to $S_{1}$ transition. For molecule III and V, the ISC process from $S_{1}$ to $T_{1}$ also lead to a significant occupation of the triplet state. The $T_{1}$ state of molecules I, III, and V decays to $S_{0}$ via the IC channel. For the rest of the molecules, the decay channels involving the triplet manifold is negligible.

The calculated rate constants for the radiative process from $S_{1}$ to $S_{0}$ of molecules I-III are much smaller than the IC rate. However, fluorescence have been experimentally detected from the $S_{1}$ state of molecules I-III. The calculated radiative transition rates from the Q band is probably underestimated, because Herzberg-Teller effects that increase the intensity of the Q bands by orders of magnitude have not been accounted for in this work. ${ }^{67}$ Fluorescence has not been observed from the $S_{1}$ state of molecules IV-VI, whereas weak fluorescence has 


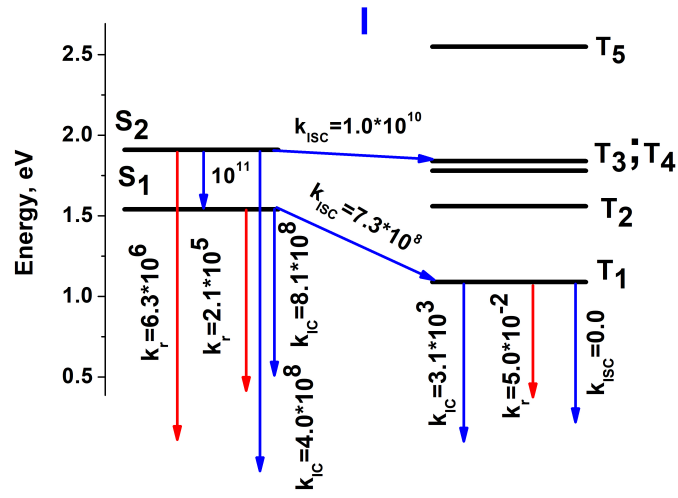

(a)

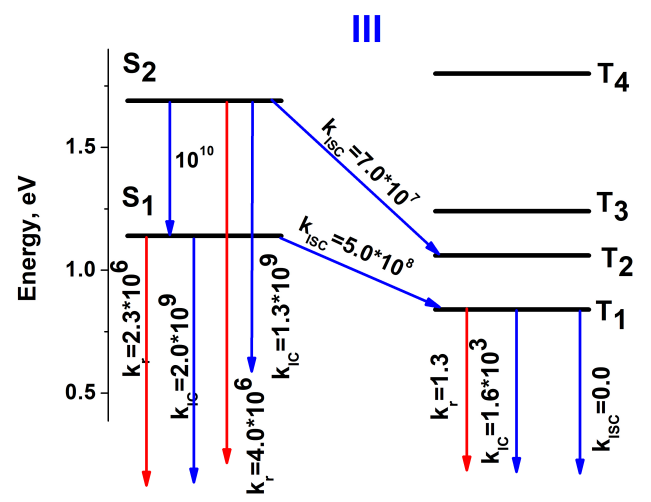

(c)

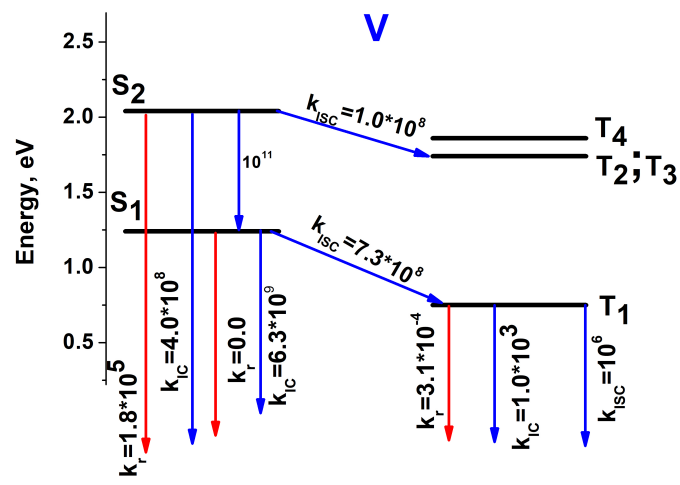

(e)

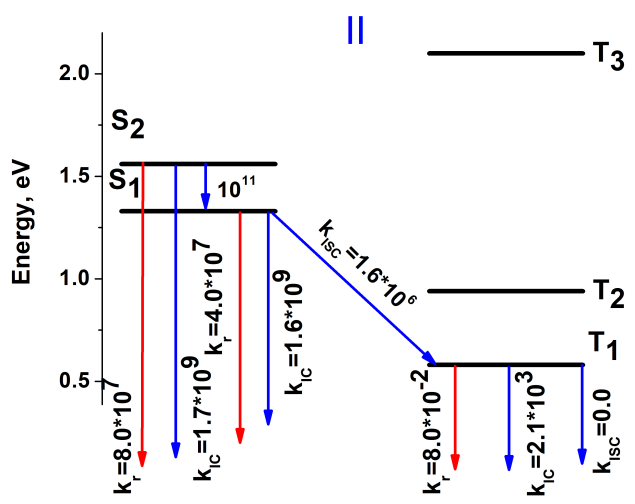

(b)

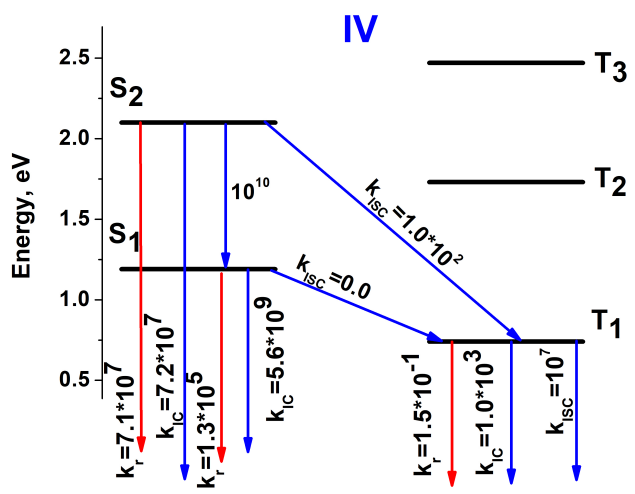

(d)

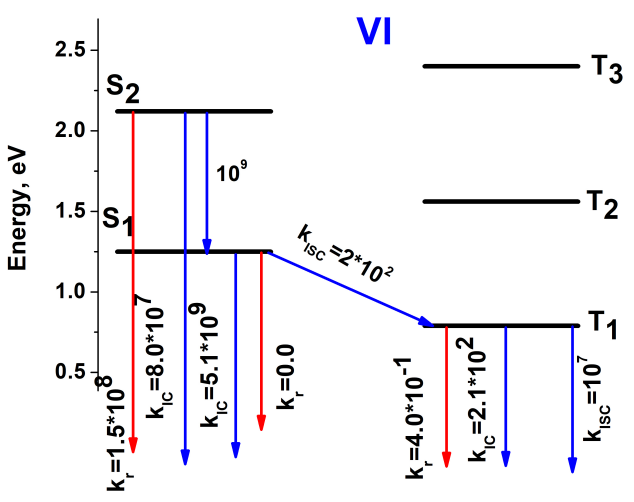

(f)

Figure 4: The rate constants (in $\mathrm{s}^{-1}$ ) of $S_{1}, S_{2}, T_{1}$ states for molecules I-VI. The red arrows represent radiative transitions, whereas the blue ones show nonradiative deactivation channels. The open ended arrows illustrate transitions to the $S_{0}$ state. 
been detected from the $S_{2}$ state of molecule IV. ${ }^{34,36}$ The calculated rate constant for the radiative transition from $S_{2}$ to $S_{0}$ of molecule VI is a factor of 30 smaller than the IC rate constant. By considering the Herzberg-Teller effect, the ratio between the two rate constants can be smaller leading to two significant decay channels and a weak fluorescence.

For molecules I-III, the fluorescence quantum yield from the $S_{1}$ state $\left(\phi\left(S_{1}\right)\right)$ is significantly larger than the fluorescence quantum yield from the $S_{2}$ state $\left(\phi\left(S_{2}\right)\right)$, because the IC process from $S_{2}$ to $S_{1}$ is very fast. The relatively small difference in the radiative and nonradiative rate constants for the decay of the $S_{1}$ state results in the observed fluorescence, especially when one considers that Herzberg-Teller effects have been omitted in the calculations. The antiaromatic molecules have very small $\phi\left(S_{1}\right)$ values, whereas the quantum yield from the $S_{2}$ state is much larger than $\phi\left(S_{1}\right)$. The large $\phi\left(S_{2}\right)$ value for molecule VI explains the detected fluorescence.

Table 5: Calculated lifetimes ( $\tau$ in $\mu$ s and ps) of the $S_{1}, S_{2}, T_{1}$ states of molecules I-VI are compared with experimental data. The calculated fluorescence quantum yield ( $\phi$ in \%) for the $S_{1}$ and $S_{2}$ states of molecules I-VI are also reported.

\begin{tabular}{lllllll}
\hline Property & I & II & III & IV & V & VI \\
\hline$\tau\left(T_{1}\right), \mu \mathrm{s}$ & 322 & 476 & 625 & 0.1 & 1 & 0.1 \\
\hline$\tau\left(T_{1}\right), \mu \mathrm{s}$ (Exp. $)^{a}$ & 14.9 & 16 & 146 & & 0.002 & \\
\hline$\tau\left(S_{1}\right), \mathrm{ps}$ & 653 & 609 & 424 & 178 & 142 & 196 \\
\hline$\tau\left(S_{1}\right), \mathrm{ps}$ (Exp.) $)^{a}$ & 2430 & 430 & 560 & & & \\
\hline$\tau\left(S_{2}\right), \mathrm{ps}$ & 87 & 10 & 421 & 98 & 9.9 & 813 \\
\hline$\tau\left(S_{2}\right), \mathrm{ps}(\text { Exp. })^{a}$ & & & & 8.7 & 30 & 110 \\
\hline$\phi\left(S_{1}\right), \%$ & 0.013 & 2.6 & 0.1 & 0.002 & 0 & 0 \\
\hline$\phi\left(S_{2}\right), \%$ & 0.005 & 0.08 & 0.04 & 0.7 & 0.0001 & 12 \\
\hline
\end{tabular}

$\bar{a}$ The experimental values were taken from the Ref. 35

The calculated lifetimes and fluorescence quantum yields are summarized in 5 . The deviations between calculated and measured lifetimes do generally not exceed one order of magnitude, implying that the calculations yield qualitatively the correct trends. The calculated lifetimes of the $\tau\left(T_{1}\right)$ state are systematically longer than obtained experimentally suggesting that the calculated $T_{1}$ excitation energies are somewhat too large. The lifetimes of the $S_{1}$ state of molecules II and III agree well with experimental data. For molecule I, the 
experimental lifetime is about four times larger than obtained in the calculations. For the $S_{2}$ state of molecules IV and VI, the calculated lifetimes are about an order of magnitude larger than the measured ones, whereas for molecule $\mathrm{V}$ the measured lifetime of the $S_{2}$ state is three times larger than the calculated one.

Table 6: Comparison of excitation energies (in eV) calculated at the XMCQDPT2 level with experimental data. Calculated and experimental decay rates (in $\mathrm{s}^{-1}$ ) of the $S_{1}, S_{2}$ and $T_{1}$ states are also compared.

\begin{tabular}{lllcccc}
\hline \hline \multirow{2}{*}{$\begin{array}{l}\text { Molecule } \\
\text { and state }\end{array}$} & \multicolumn{2}{c}{ Excitation energy } & \multicolumn{4}{c}{ Decay rate of } \\
Exp. & Calc & $S_{1}$ or $S_{2}$ (Exp.) & $S_{1}$ or $S_{2}$ (Calc.) & $T_{1}$ (Exp.) & $T_{1}$ (Calc.) \\
\hline I $S_{1}$ & 1.74 & 1.54 & $4 \cdot 10^{8}$ & $1 \cdot 10^{9}$ & $7 \cdot 10^{4}$ & $3.1 \cdot 10^{3}$ \\
II $S_{1}$ & 1.56 & 1.34 & $2 \cdot 10^{9}$ & $2 \cdot 10^{9}$ & $6 \cdot 10^{4}$ & $2.1 \cdot 10$ \\
III $S_{1}$ & 1.34 & 1.14 & $2 \cdot 10^{9}$ & $1 \cdot 10^{10}$ & $7 \cdot 10^{3}$ & $1.6 \cdot 10^{4}$ \\
IV $S_{2}$ & 2.25 & 2.10 & $1 \cdot 10^{11}$ & $5 \cdot 10^{10}$ & - & $1.0 \cdot 10^{7}$ \\
V $S_{2}$ & 2.24 & 2.05 & $3 \cdot 10^{10}$ & $1 \cdot 10^{11}$ & $5 \cdot 10^{8}$ & $1.0 \cdot 10^{6}$ \\
VI $S_{2}$ & 2.08 & 2.12 & $1 \cdot 10^{10}$ & $1 \cdot 10^{9}$ & - & $1.0 \cdot 10^{7}$ \\
\hline \hline
\end{tabular}

The calculated decay rates of the lowest singlet and triplet states are compared to experimental data in 6 . The obtained values for the singlet states are in good agreement with experimental values, since they deviate by less than one order of magnitude. The calculated decay rates of the $T_{1}$ states are generally one to three orders of magnitude too small as compared to experimental data. For molecule III, the calculated and measured decay rates of the $T_{1}$ state agree well. Singlet excitation energies calculated at the XMC-QDPT2 level are about $0.2 \mathrm{eV}$ smaller than the experimental values. Excitation energies of the lowest triplet state have not been measured for the studied molecules.

\section{Summary and conclusions}

Magnetic and optical properties have been studied computationally for a set of six expanded porphyrins. The aromatic character was assessed by analysing the magnetically induced current density of the molecules. By scrutinizing the integrated current strength susceptibility for selected bonds, the aromatic pathways have been identified. Sapphyrin (I), cyclo[6]pyrrole 
(II) and rubyrin (III) are aromatic sustaining net diatropic ring currents, whose strengths are 24-30 nA/T at the B3LYP level. The ring-current strengths are of about the same size as for free-base porphyrin. Organarin (IV), rosarin (V) and amethyrin (VI) are antiaromatic sustaining net paratropic ring currents of $-23 \mathrm{nA} / \mathrm{T}$ to $-30 \mathrm{nA} / \mathrm{T}$ at the MP2 level of theory. The current divides into two branches at each pyrrolic ring suggesting that all $\pi$-electrons participate in the aromatic pathway.

For the aromatic molecules, the ring current flows preferably via the $\beta$ carbons of the pyrrolic rings with an inner hydrogen, while it splits nearly equally at the other pyrrolic rings though with a slightly stronger current via the nitrogen.

For the antiaromatic molecules, strong paratropic currents flow via the nitrogen moiety regardless whether it has an inner hydrogen or not. The strength of the ring current passing the $\beta$ carbons is between $-1.2 \mathrm{nA} / \mathrm{T}$ and $-8.6 \mathrm{nA} / \mathrm{T}$. Thus, on the average a small fraction of about $18 \%$ of the ring current takes the outer route at the pyrrolic rings.

Calculations at different levels of theory show that for the aromatic molecules about about the same ring-current strengths are obtained at the DFT level using different functionals. The current strengths obtained at the HF and MP2 level also agree well with those calculated at the DFT levels. However, the ring current strength calculated for molecule I at the MP2 level is for some unknown reason 18-25 nA/T larger than obtained at the other levels of theory. The calculated ring-current strengths of the antiaromatic molecules depend on the amount of HF exchange in the functional. The BHLYP functional with $50 \% \mathrm{HF}$ exchange yields ring-current strengths in good agreement with MP2 values. Functionals with less HF exchange yield stronger paratropic ring currents. HF calculations seem to underestimate the strength of the paratropic ring current of the antiaromatic porphyrinoids.

Singlet and triplet excitation energies have been calculated using the XMC-QDPT2 method. Excitation energies have also been calculated at the CC2 and B3LYP levels. For the aromatic molecules, CC2 and B3LYP calculations yield somewhat larger excitation energies than obtained at the XMC-QDPT2 level. For the antiaromatic molecules, the $S_{1}$ to 
$S_{0}$ transition is a magnetic dipole allowed transition, which explains why the molecules are antiaromatic sustaining strong paratropic ring currents. The CC2 energies for the $\mathrm{S}_{1}$ state of the antiaromatic molecules agree well with the XMC-QDPT2 ones, whereas B3LYP slightly underestimates them. The $\mathrm{CC} 2$ excitation energies of the $S_{2}$ state are much larger than the XMC-QDPT2 ones indicating a significant double excitation character of the $S_{2}$ state of the antiaromatic molecules. The calculated spin-orbit coupling interactions reveal a strong coupling between the $T_{1}$ state and the $S_{0}$ ground state for the antiaromatic molecules IV-VI, while the spin-orbit coupling between these states vanishes for the aromatic molecules (I-III).

Rate constants for radiative and nonradiative processes have been obtained by calculating spin-orbit coupling matrix elements, the oscillator strengths, and excitation energies at the XMC-QDPT2 level. Nonadiabatic coupling matrix elements were calculated at the semiempirical INDO level. The XMC-QDPT2 calculations yield singlet excitation energies that are about $0.2 \mathrm{eV}$ smaller than experimental values. The calculated rate constants show that the excitation-energy decay process is mainly dominated by deactivation along IC channels. The triplet manifold participates in the deactivation process for molecules I, III and V. For the aromatic molecules, the radiative transition rate from the $S_{1}$ state is not much smaller than the IC rate, which explains the observed fluorescence. The $S_{1}$ state of the antiaromatic molecules is dark, whereas fluorescence has been detected from the $S_{2}$ state of molecule VI. The calculations show that fluoresence from the $S_{2}$ state is a significant decay channel, since the obtained radiative rate costant for the $S_{2}$ to $S_{0}$ transition is of about the same magnitude as the one calculated for the IC processes. Calculated and measured decay rates of the $S_{1}$ and $S_{2}$ states agree within an order of magnitude with experimental data. For $T_{1}$, the deviations are somewhat larger.

The present study shows that accurate rate constants can be calculated for excitationenergy decay channels via radiative and nonradative processes by using high-level electronic structure methods such as the expanded multi-configurational quasi-degenerate perturbation theory (XMC-DPT2) approach. The obtained optical properties depend on the aromatic 
character of the expanded porphyrins, which have been investigated in detail by performing explicit current-density and current-strength calculations using the GIMIC method.

\section{Acknowledgement}

This research has been supported by the Academy of Finland through projects (275845 and 297304) and by the Magnus Ehrnrooth foundation. CSC - IT Center for Science, Finland and the Finnish Grid and Cloud Infrastructure (FGCI) are acknowledged for computer time. H. F. thanks the Norwegian Research Council through the CoE Hylleraas Centre for Quantum Molecular Sciences (Grant No. 262695 and 231571/F20) for support. This work has received support from the Norwegian Supercomputing Program (NOTUR) through a grant of computer time (Grant No. NN4654K). The research was carried out at the expense of the grant from Russian Science Foundation (project No. 17-73-20012). Also, the research is carried out at Tomsk Polytechnic University within the framework of Tomsk Polytechnic University Competitiveness Enhancement Program grant.

\section{Supporting Information Available}




\section{References}

(1) Paolesse, R.; Nardis, S.; Monti, D.; Stefanelli, M.; Di Natale, C. Porphyrinoids for Chemical Sensor Applications. Chem. Rev. 2017, 117, 2517-2583.

(2) Reddy, J. S.; Anand, V. G. Planar Meso Pentafluorophenyl Core Modified Isophlorins. J. Am. Chem. Soc. 2008, 130, 3718-3719.

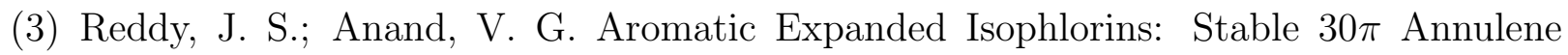
Analogues with Diverse Structural Features. J. Am. Chem. Soc. 2009, 131, 1543315439.

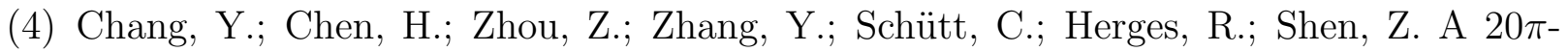
Electron Heteroporphyrin Containing a Thienopyrrole Unit. Angew. Chem. Int. Ed. 2012, 51, 12801-12805.

(5) Berlicka, A.; Dutka, P.; Szterenberg, L.; Latos-Grażyński, L. Towards True Carbaporphyrinoids: Synthesis of 21-Carba-23-thiaporphyrin. Angew. Chem. Int. Ed. 2014, 53, $4885-4889$.

(6) Berlin K., Carbaporphyrins. Angew. Chem. Int. Ed. 1996, 35, 1820-1822.

(7) Lash, T. D.; Gilot, G. C.; AbuSalim, D. I. Tropone-Fused Carbaporphyrins. J. Org. Chem. 2014, 79, 9704-9716.

(8) Shin, J.-Y.; Kim, K. S.; Yoon, M.-C.; Lim, J. M.; Yoon, Z. S.; Osuka, A.; Kim, D. Aromaticity and Photophysical Properties of Various Topology-Controlled Expanded Porphyrins. Chem. Soc. Rev. 2010, 39, 2751-2767.

(9) Osuka, A.; Saito, S. Expanded Porphyrins and Aromaticity. Chem. Commun. 2011, 47, 4330-4339.

(10) Breslow, R. Antiaromaticity. Acc. Chem. Res. 1973, 6, 393-398. 
(11) Breslow, R. Novel Aromatic and Antiaromatic Systems. Chem. Rec. 2014, 14, 11741182.

(12) Shin, J.-Y.; Yamada, T.; Yoshikawa, H.; Awaga, K.; Shinokubo, H. An Antiaromatic Electrode-Active Material Enabling High Capacity and Stable Performance of Rechargeable Batteries. Angew. Chem. Int. Ed. 2014, 53, 3096-3101.

(13) Fujii, S.; Marqués-González, S.; Shin, J.; Shinokubo, H.; Masuda, T.; Nishino, T.; Arasu, N. P.; Vázquez, H.; Kiguchi, M. Highly-Conducting Molecular Circuits Based on Antiaromaticity. Nat. Commun. 2017, 8, 15984-(1-8).

(14) Steiner, E.; Fowler, P. W. Diamagnetic and Paramagnetic Ring Currents in Expanded Porphyrins. Org. Biomol. Chem. 2004, 2, 34-37.

(15) Steiner, E.; Fowler, P. W.; Soncini, A.; Jenneskens, L. W. Current-Density Maps as Probes of Aromaticity: Global and Clar $\pi$ Ring Currents in Totally Resonant Polycyclic Aromatic Hydrocarbons. Faraday Discuss. 2007, 135, 309-323.

(16) Steiner, E.; Soncini, A.; Fowler, P. W. Ring Currents in the Porphyrins: $\pi$ Shielding, Delocalisation Pathways and the Central Cation. Org. Biomol. Chem. 2005, 4, 40534059.

(17) Fliegl, H.; Sundholm, D.; Taubert, S.; Pichierri, F. Aromatic Pathways in Twisted Hexaphyrins. J. Phys. Chem. A 2010, 114, 7153-7161.

(18) Fliegl, H.; Sundholm, D.; Pichierri, F. Aromatic Pathways in Mono- and Bisphosphorous Singly Möbius Twisted [28] and [30]Hexaphyrins. Phys. Chem. Chem. Phys. 2011, 13, 20659-20665.

(19) Fliegl, H.; Sundholm, D. Aromatic Pathways of Porphins, Chlorins and Bacteriochlorins. J. Org. Chem. 2012, 77, 3408-3414. 
(20) Fliegl, H.; Özcan, N.; Mera-Adasme, R.; Pichierri, F.; Jusélius, J.; Sundholm, D. Aromatic Pathways in Thieno-Bridged Porphyrins: Understanding the Influence of the Direction of the Thiophene Ring on the Aromatic Character. Mol. Phys. 2013, 111, $1364-1372$.

(21) Valiev, R. R.; Cherepanov, V. N. The Influence of Benzene Rings on Aromatic Pathways in the Porphyrins. Int. J. Quant. Chem. 2013, 113, 2563-2567.

(22) Valiev, R. R.; Fliegl, H.; Sundholm, D. Predicting the Degree of Aromaticity of Novel Carbaporphyrinoids. Phys. Chem. Chem. Phys. 2015, 17, 14215-14222.

(23) Benkyi, I.; Fliegl, H.; Valiev, R. R.; Sundholm, D. New Insights on Aromatic Pathways of Carbachlorins and Carbaporphyrins Based on Calculations of Magnetically Induced Current Densities. Phys. Chem. Chem. Phys. 2016, 18, 11932-11941.

(24) Valiev, R. R.; Fliegl, H.; Sundholm, D. Aromatic Pathways in Carbathiaporphyrins. J. Phys. Chem. A 2015, 119, 1201-1207.

(25) Valiev, R. R.; Fliegl, H.; Sundholm, D. The Aromatic Character of Thienopyrrole-

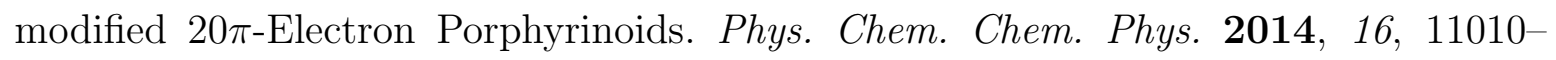
11016.

(26) Valiev, R. R.; Fliegl, H.; Sundholm, D. New Insights into Magnetically Induced Current Pathways and Optical Properties of Isophlorins. J. Phys. Chem. A 2013, 117, 90629068.

(27) Fliegl, H.; Pichierri, F.; Sundholm, D. Antiaromatic Character of $16 \pi$ Electron Octaethylporphyrins: Magnetically Induced Ring Currents from DFT-GIMIC Calculations. J. Phys. Chem. A 2015, 119, 2344-2350.

(28) Fliegl, H.; Valiev, R. R.; Pichierri, F.; Sundholm, D. Theoretical Studies as A Tool for 
Understanding the Aromatic Character of Porphyrinoid Compounds. Chem. Modell. 2018, 14, 1-42.

(29) Valiev, R. R.; Fliegl, H.; Sundholm, D. Closed-Shell Paramagnetic Porphyrinoids. Chem. Comm. 2017, 53, 9866-9869.

(30) Peeks, M. D.; Claridge, T. D. W.; Anderson, H. L. Aromatic and Antiaromatic Ring Currents in a Molecular Nanoring. Nature 2017, 541, 200-203.

(31) K. K. Lange and E. I. Tellgren and M. R. Hoffmann and T. Helgaker, A Paramagnetic Bonding Mechanism for Diatomics in Strong Magnetic Fields. Science 2012, 337, 327331.

(32) Tellgren, E. I.; Helgaker, T.; Soncini, A. Non-Perturbative Magnetic Phenomena in Closed-Shell Paramagnetic Molecules. Phys. Chem. Chem. Phys. 2009, 11, 5489-5498.

(33) Valiev, R. R.; Fliegl, H.; Sundholm, D. Optical and Magnetic Properties of Antiaromatic Porphyrinoids. Phys. Chem. Chem. Phys. 2017, 19, 25979-25988.

(34) Yoon, Z. S.; Cho, D.-G.; Kim, K. S.; Sessler, J. L.; Kim, D. Nonlinear Optical Properties as a Guide to Aromaticity in Congeneric Pentapyrrolic Expanded Porphyrins: Pentaphyrin, Sapphyrin, Isosmaragdyrin, and Orangarin. J. Am. Chem. Soc. 2008, 130, 6930-6931.

(35) Cho, S.; Yoon, Z. S.; Kim, K. S.; Yoon, M.-C.; Cho, D.-G.; Sessler, J. L.; Kim, D. Defining Spectroscopic Features of Heteroannulenic Antiaromatic Porphyrinoids. J. Phys. Chem. Lett. 2010, 1, 895-900.

(36) Yoon, M.-C.; Cho, S.; Suzuki, M.; Osuka, A.; Kim, D. Aromatic Versus Antiaromatic Effect on Photophysical Properties of Conformationally Locked trans-Vinylene-Bridged Hexaphyrins. J. Am. Chem. Soc. 2009, 131, 7360-7367. 
(37) Valiev, R. R.; Cherepanov, V. N.; Artyukhov, V. Y.; Sundholm, D. Computational Studies of Photophysical Properties of Porphin, Tetraphenylporphyrin and Tetrabenzoporphyrin. Phys. Chem. Chem. Phys. 2012, 14, 11508-11517.

(38) Baryshnikov, G. V.; Valiev, R. R.; Karaush, N. N.; Minaeva, V. A.; Sinelnikov, A. N.; Pedersen, S. K.; Pittelkow, M.; Minaev, B. F.; Ågren, H. Benzoannelated Aza-, Oxaand Azaoxa[8]circulenes as Promising Blue Organic Emitters. Phys. Chem. Chem. Phys. 2016, 18, 28040-28051.

(39) Baryshnikov, G. V.; Valiev, R. R.; Minaev, B. F.; Ågren, H. A Computational Study of Aromaticity and Photophysical Properties of Unsymmetrical Azatrioxa[8]circulenes. New J. Chem. 2017, 41, 717-2723.

(40) Baryshnikov, G. V.; Valiev, R. R.; Minaev, B. F.; Ågren, H. Substituent-Sensitive Fluorescence of Sequentially N-Alkylated Tetrabenzotetraaza[8]circulenes. New J. Chem. 2017, 41, 7621-7625.

(41) Becke, A. D. Density-Functional Thermochemistry. III. The Role of Exact Exchange. J. Chem. Phys. 1993, 98, 5648-5652.

(42) Lee, C.; Yang, W.; Parr, R. G. Development of the Colle-Salvetti Correlation-Energy Formula into a Functional of the Electron Density. Phys. Rev. B 1988, 37, 785-789.

(43) Schäfer, A.; Horn, H.; Ahlrichs, R. Fully Optimized Contracted Gaussian-Basis Sets for Atoms Li to Kr. J. Chem. Phys. 1992, 97, 2571-2577.

(44) Weigend, F.; Ahlrichs, R. Balanced Basis Sets of Split Valence, Triple Zeta Valence and Quadruple Zeta Valence Quality For H to Rn: Design and Assessment of Accuracy. Phys. Chem. Chem. Phys. 2005, 7, 3297-3305.

(45) Ahlrichs, R.; Bär, M.; Häser, M.; Horn, H.; Kölmel, C. Electronic Structure Calculations 
on Workstation Computers: The Program System TURBOMOLE. Chem. Phys. Letters 1989, 162, 165-169, current version: see http://www.turbomole.com.

(46) Furche, F.; Ahlrichs, R.; Hättig, C.; Klopper, W.; Sierka, M.; Weigend, F. Turbomole. WIREs Comput. Mol. Sci. 2014, 4, 91-100.

(47) Casademont-Reig, I.; Woller, T.; Contreras-Garcia, J.; Alonso, M.; TorrentSucarrat, M.; Matito, E. New Electron Delocalization Tools to Describe the Aromaticity in Porphyrinoids. Phys. Chem. Chem. Phys. 2018, 20, 2787-2796.

(48) Torrent-Sucarrat, M.; Navarro, S.; Cossío, F. P.; Anglada, J. M.; Luis, J. M. Relevance of the DFT Method to Study Expanded Porphyrins with Different Topologies. J. Comp. Chem. 38, 2819-2828.

(49) Szczepanik, D. W.; Sol, M.; Andrzejak, M.; Paweek, B.; Dominikowska, J.; Kukuka, M.; Dyduch, K.; Krygowski, T. M.; Szatylowicz, H. The Role of the Long-Range Exchange Corrections in the Description of Electron Delocalization in Aromatic Species. J. Comp. Chem. 2017, 38, 1640-1654.

(50) Granovsky, A. A. Extended Multi-Configuration Quasi-Degenerate Perturbation Theory: The New Approach to Multi-State Multi-Reference Perturbation Theory. J. Chem. Phys. 2011, 134, 214113.

(51) Andersson, K.; Malmqvist, P. A.; Roos, B. O. 2nd-Order Perturbation-Theory with a Complete Active Space Self-Consistent Field Reference Function. J. Chem. Phys. 1992, $96,1218-1226$.

(52) Angeli, C.; Cimiraglia, R.; Malrieu, J. Introduction of n-Electron Valence States for Multireference Perturbation Theory. J. Chem. Phys. 2001, 114, 10252-10264.

(53) Granovsky, A. A. Firefly, version 8.0.0, http://classic.chem.msu.su/gran/firefly/index.html. 
(54) Christiansen, O.; Koch, H.; Jørgensen, P. The 2nd-order Approximate Coupled-Cluster Singles and Doubles Model CC2. Chem. Phys. Letters 1995, 243, 409-418.

(55) Hättig, C.; Weigend, F. CC2 Excitation Energy Calculations on Large Molecules Using the Resolution of the Identity Approximation. J. Chem. Phys. 2000, 113, 5154-5161.

(56) Köhn, A.; Hättig, C. Analytic Gradients for Excited States in the Coupled-Cluster Model CC2 Employing the Resolution-of-the-Identity Approximation. J. Chem. Phys. 2003, 119, 5021-5036.

(57) Hättig, C. Structure Optimizations for Excited States with Correlated Second-Order Methods: CC2 and ADC(2). Adv. Quantum Chem. 2005, 50, 37-60.

(58) Ahlrichs, R.; Bär, M.; Häser, M.; Horn, H.; Kölmel, C. Electronic Structure Calculations on Workstation Computers: The Program System TURBOMOLE. Chem. Phys. Letters 1989, 162, 165-169, current version: see http://www.turbomole.com.

(59) Send, R.; Kaila, V. R. I.; Sundholm, D. Reduction of the Virtual Space for CoupledCluster Excitation Energies of Large Molecules and Embedded Systems. J. Chem. Phys. 2011, 134, 214114.

(60) Head-Gordon, M.; Rico, R. J.; Manabu, O.; Lee, T. J. A Doubles Correction to Electronic Excited States from Configuration Interaction in the Space of Single Substitutions. Chem. Phys. Letters 1994, 219, 21-29.

(61) Casida, M.; Huix-Rotllant, M. Progress in Time-Dependent Density-Functional Theory. Annu. Rev. Phys. Chem. 2012, 63, 287-323.

(62) Furche, F.; Ahlrichs, R. Adiabatic Time-Dependent Density Functional Methods for Excited State Properties. J. Chem. Phys. 2002, 117, 7433-7447.

(63) Schmidt, M. W.; Baldridge, K. K.; Boatz, J. A.; Elbert, S. T.; Gordon, M. S.; 
Jensen, J. H.; Koseki, S.; Matsunaga, N.; Nguyen, K. A.; Su, S. et al. General Atomic and Molecular Electronic Structure System. J. Comp. Chem. 1993, 14, 1347-1363.

(64) Strickler, S. J.; Berg, R. A. Relationship between Absorption Intensity and Fluorescence Lifetime of Molecules. J. Chem. Phys. 1962, 37, 814-822.

(65) Artyukhov, V. Y.; Kopylova, T. N.; Samsonova, L. G.; Selivanov, N. I.; Plotnikov, V. G.; Sazhnikov, V. A.; Khlebunov, A. A.; Mayer, G. V.; Alfimov, M. V. A Combined Theoretical and Experimental Study on Molecular Photonics. Russ. Phys. J. 2008, 51, $1097-1111$.

(66) Plotnikov, V. G. Regularities of the Processes of Radiationless Conversion in Polyatomic Molecules. Int. J. Quant. Chem. 1979, 16, 527-541.

(67) Santoro, F.; Lami, A.; Improta, R.; Bloino, J.; Barone, V. Effective Method for the Computation of Optical Spectra of Large Molecules at Finite Temperature Including the Duschinsky and Herzberg-Teller Effect: The $Q_{x}$ Band of Porphyrin as a Case Study. J. Chem. Phys. 2008, 128, 224311.

(68) McGlynn, S. P.; Azumi, T.; Kinoshita, M. Molecular spectroscopy of the triplet state; Englewood Cliffs: New Jersey, 1969.

(69) Jusélius, J.; Sundholm, D.; Gauss, J. Calculation of Current Densities Using GaugeIncluding Atomic Orbitals. J. Chem. Phys. 2004, 121, 3952-3963.

(70) Taubert, S.; Sundholm, D.; Jusélius, J. Calculation of Spin-Current Densities Using Gauge-Including Atomic Orbitals. J. Chem. Phys. 2011, 134, 054123:1-12.

(71) Fliegl, H.; Taubert, S.; Lehtonen, O.; Sundholm, D. The Gauge Including Magnetically Induced Current Method. Phys. Chem. Chem. Phys. 2011, 13, 20500-20518.

(72) Sundholm, D.; Fliegl, H.; Berger, R. J. Calculations of Magnetically Induced Current Densities: Theory and Applications. WIREs Comput. Mol. Sci. 2016, 6, 639-678. 
(73) Schäfer, A.; Huber, C.; Ahlrichs, R. Fully Optimized Contracted Gaussian-Basis Sets of Triple Zeta Valence Quality for Atoms Li to Kr. J. Chem. Phys. 1994, 100, 5829-5835.

(74) Ditchfield, R. Self-Consistent Perturbation-Theory of Diamagnetism 1. Gauge-Invariant LCAO Method For NMR Chemical-Shifts. Mol. Phys. 1974, 27, 789-807.

(75) Wolinski, K.; Hinton, J. F.; Pulay, P. Efficient Implementation of the GaugeIndependent Atomic Orbital Method for NMR Chemical Shift Calculations. J. Am. Chem. Soc. 1990, 112, 8251-8260.

(76) Häser, M.; Ahlrichs, R.; Baron, H. P.; Weis, P.; Horn, H. Direct Computation of 2ndOrder SCF Properties of Large Molecules on Workstation Computers with an Application to Large Carbon Clusters. Theoret. Chim. Acta 1992, 83, 455-470.

(77) Kollwitz, M.; Häser, M.; Gauss, J. Non-Abelian Point Group Symmetry in Direct Second-Order Many-Body Perturbation Theory Calculations of NMR Chemical Shifts. J. Chem. Phys. 1998, 108, 8295-8301.

(78) Becke, A. D. A New Mixing of Hartree-Fock and Local Density-Functional Theories. J. Chem. Phys. 1993, 98, 1372-1377.

(79) Perdew, J. P.; Ernzerhof, M.; Burke, K. Rationale for Mixing Exact Exchange with Density Functional Approximations. J. Chem. Phys. 1996, 105, 9982-9985.

(80) Tao, J.; Perdew, J. P.; Staroverov, V. N.; Scuseria, G. E. Climbing the Density Functional Ladder: Nonempirical Meta-Generalized Gradient Approximation Designed for Molecules and Solids. Phys. Rev. Letters 2003, 91, 146401 1-4.

(81) Staroverov, V. N.; Scuseria, G. E.; Tao, J.; Perdew, J. P. Comparative Assessment of a New Nonempirical Density Functional: Molecules and Hydrogen-Bonded Complexes. J. Chem. Phys. 2003, 119, 12129. 
(82) Zhao, Y.; Truhlar, D. G. The M06 Suite of Density Functionals for Main Group Thermochemistry, Thermochemical Kinetics, Noncovalent Interactions, Excited States, and Transition Elements: Two New Functionals and Systematic Testing of Four M06-Class Functionals and 12 Other Functionals. Theo. Chem. Acc. 2008, 120, 215-241.

(83) Reiter, K.; Mack, F.; Weigend, F. Calculation of Magnetic Shielding Constants with Meta-GGA Functionals Employing the Multipole-Accelerated Resolution of the Identity: Implementation and Assessment of Accuracy and Efficiency. J. Chem. Theory Comput. 2018, 14, 191-197.

(84) Kumar, C.; Fliegl, H.; Sundholm, D. The Relation Between Ring Currents and Hydrogenation Enthalpies for Assessing the Degree of Aromaticity. J. Phys. Chem. A 2017, 121, 7282-7289.

(85) G. Baryshnikov, B. M.; Ågren, H. Theory and Calculation of the Phosphorescence Phenomenon. Chem. Rev. 2017, 117, 6500-6537. 